\title{
Electromagnetic (Darwin) Model for Three-Dimensional Perturbative Particle Simulation of High Intensity Beams
}

\author{
W. Wei-li Lee, Edward Startsev, Hong Qin, and Ronald C. Davidson \\ Plasma Physics Laboratory, Princeton University, Princeton, NJ 08543, USA
}

\begin{abstract}
A nonlinear perturbative $(\delta f)$ electromagnetic particle simulation scheme has been developed for studying the propagation of intense charged particle beams in highintensity accelerators and transport systems. The scheme is based on the Darwin approximation of Ampere's law, in which the transverse displacement current is neglected, resulting in the elimination of high-frequency transverse electromagnetic effects and, consequently, the associated numerical restrictions in the simulations. In this paper, a novel approach of replacing the mechanical momentum, $\mathbf{p}$, by the canonical momentum, $\mathbf{P}=\mathbf{p}+q \mathbf{A} / c$, in the equations of motion is used for the purpose of eliminating the numerically troublesome $\partial \mathbf{A} / \partial t$ term. The present scheme, cast in the $\delta f$ formalism, is ideal for studying twostream and filamentation instabilities, which may cause deterioration of the beam quality in heavy ion fusion drivers and the fusion chamber.
\end{abstract}

\section{INTRODUCTION}

A nonlinear perturbative $(\delta f)$ electromagnetic particle simulation scheme has been developed for studying the propagation of intense charged particle beams in highintensity accelerators and transport systems. The scheme is based on the Darwin approximation of Ampere's law, in which the transverse inductive electric field is neglected, resulting in the elimination of light waves as well as the associated numerical restrictions from the simulations. However, as noted in the past [1], the presence of the time derivative of the vector potential, $\partial \mathbf{A} / \partial t$, in the equations of motion for the Darwin model can cause numerical instabilities. To circumvent this difficulty, procedures involving the removal of $\partial \mathbf{A} / \partial t$ in the equations of motion have been developed, and the Darwin model has been successfully used in particle simulations for studying electromagnetic perturbations in plasmas, such as Weibel instabilities [1,2], whistler and magnetosonic waves [3] and shear-Alfvén waves [4]. Other methods involving the calculation of the transverse inductive electric field have also been developed $[5,6]$. In this paper, we adopt a procedure similar to the Hamiltonian formulation suggested by Nielson and Lewis [1] in which the mechanical momentum, $\mathbf{p}=\gamma m \mathbf{v}$, is replaced by the canonical momentum, $\mathbf{P}=\mathbf{p}+(q / c) \mathbf{A}$, as a phase-space variable so as to eliminate the troublesome $\partial \mathbf{A} / \partial t$ term, where $q$ is the charge, $c$ is the speed of light in vacuo, and $m$ is the rest mass. The advantage of using the canonical momentum has long been recognized in magnetic fusion research for test particle transport [7] and for gyrokinetic theory [8, 9]. In order to take advantage of the recent developments in perturbative particle simulations $[10,11]$, the present scheme is also cast into the $\delta f\left(\equiv F-F_{0}\right)$ formalism, where $F$ is the distribution function in phase space, and $F_{0}$ is the equilibrium distribution. As a result, the simulation plasma has minimal numerical noise, and also provides us with the ability to easily access both linear and nonlinear regimes for the physics of interest. Since the high-frequency waves associated with radiation fields are absent from the simulations, we can use the scheme of adiabatic particle pushing [12], for which the electrons are advanced more often, and with smaller time steps, than those for the ions and field equations so as to compensate for the mass ratio disparities for different charge species. The scheme is ideal for studying two-stream[12] and filamentation [13] instabilities, which may cause deterioration of the beam quality in the heavy ion fusion driver and fusion chamber.

\section{DARWIN MODEL FOR RELATIVISTIC BEAMS}

Here, we take a different approach by eliminating the time derivative of the transverse electric field in the equation of motion for the particles through the use of the canonical momentum. The relativistic form of the Vlasov equation describing the propagation of an intense particle beam with narrow momentum spread through a smoothfocusing transverse external focusing field can be expressed as [12]

$\frac{\partial F}{\partial t}+\mathbf{v} \cdot \frac{\partial F}{\partial \mathbf{x}}+\left[-\gamma_{b} m \omega_{\beta}^{2} \mathbf{x}_{\perp}+q\left(\mathbf{E}+\frac{1}{c} \mathbf{v} \times \mathbf{B}\right)\right] \cdot \frac{\partial F}{\partial \mathbf{p}}=0$,

where $\mathbf{v}=\mathbf{p} / \gamma m, \gamma_{b}=1 / \sqrt{1-\beta_{b}^{2}}$ and $\beta_{b}=<v_{z}>/ c$, $\left\langle v_{z}\right\rangle$ is the average axial velocity, $\mathbf{x}_{\perp}$ is the perpendicular displacement, $z$ is the direction of beam propagation, and $\omega_{\beta}=$ const. is the effective applied betatron frequency for transverse (perpendicular) oscillations. In terms of longitudinal $(L)$ and transverse $(T)$ quantities relative to the direction of wave propagation, the reduced Maxwell's equations for the Darwin model can be expressed as $\nabla \cdot \mathbf{E}^{L}=4 \pi \rho, \nabla \times \mathbf{B}=(4 \pi / c) \mathbf{J}^{T}$, and $\nabla \times \mathbf{E}^{T}=-(1 / c) \partial \mathbf{B} / \partial t$. For $J_{z} \gg J_{\perp}$, the transverse current is defined as

$$
\mathbf{J}^{T}=J_{z} \hat{\mathbf{z}}+\frac{1}{4 \pi} \frac{\partial \mathbf{E}^{L}}{\partial t}
$$


which in Fourier k-space representation yields

$$
J_{z}^{T}=J_{z}\left(1-\frac{k_{z}^{2}}{k^{2}}\right), \quad \mathbf{J}_{\perp}^{T}=-\frac{k_{z} J_{z}}{k^{2}} \mathbf{k}_{\perp} .
$$

Using the Coulomb gauge with $\nabla \cdot \mathbf{A}=0$ for $\mathbf{B}=\nabla \times \mathbf{A}$, Ampere's law is then simplified to become

$$
\nabla^{2} A_{z}=-\frac{4 \pi}{c} J_{z}^{T}, \quad \nabla^{2} \mathbf{A}_{\perp}=-\frac{4 \pi}{c} \mathbf{J}_{\perp}^{T},
$$

where

$$
J_{z}=q \int F v_{z} d \mathbf{p}
$$

Likewise, we obtain

$$
\mathbf{E}^{T}=-\frac{1}{c} \frac{\partial A_{z}}{\partial t} \hat{\mathbf{e}}_{z}-\frac{1}{c} \frac{\partial \mathbf{A}_{\perp}}{\partial t},
$$

and Poisson's equation,

$$
\nabla^{2} \Phi=-4 \pi q \int F d \mathbf{p}
$$

remains unchanged. The nonlinear Vlasov equation in the Darwin approximation for $v_{z} \gg v_{\perp}$ becomes

$\frac{\partial F}{\partial t}+\mathbf{v}_{\perp} \cdot \frac{\partial F}{\partial \mathbf{x}_{\perp}}+v_{z} \frac{\partial F}{\partial z}-\left[\gamma_{b} m \omega_{\beta}^{2} \mathbf{x}_{\perp}+q \nabla_{\perp}\left(\Phi-\frac{v_{z}}{c} A_{z}\right)\right.$ $\left.+\frac{q}{c}\left(\frac{\partial \mathbf{A}_{\perp}}{\partial t}+v_{z} \frac{\partial \mathbf{A}_{\perp}}{\partial z}\right)\right] \cdot \frac{\partial F}{\partial \mathbf{p}_{\perp}}-q\left(\frac{\partial \Phi}{\partial z}+\frac{1}{c} \frac{\partial A_{z}}{\partial t}\right) \frac{\partial F}{\partial p_{z}}=0$.

Thus, the only difference between the electromagnetostatic model [12] and the present Darwin model is the appearance of the terms $\partial \mathbf{A}_{\perp} / \partial t+v_{z} \partial \mathbf{A}_{\perp} / \partial z$ and $\partial A_{z} / \partial t$. [For example, the latter can become important for $\left(\omega / k v_{z}\right)\left(v_{z} / c\right)^{2} \sim O(1)$, for $A_{z} \simeq \beta_{b} \Phi$.] The $\mathbf{J}^{T}$ term in Eq. (2) can be evaluated by first taking the time derivative of Poisson's equation and by substituting the density moment of Eq. (4) to obtain

$$
\nabla^{2} \frac{\partial \Phi}{\partial t}=4 \pi q \frac{\partial}{\partial z} \int v_{z} F d \mathbf{p}
$$

The transverse part of the current can be evaluated from

$$
\mathbf{J}^{T}=J_{z} \hat{\mathbf{z}}-(1 / 4 \pi) \nabla \partial \Phi / \partial t .
$$

\section{DARWIN MODEL FOR PARTICLE SIMULATIONS}

As discussed earlier, the new $\partial A_{z} / \partial t$ and $\partial \mathbf{A}_{\perp} / \partial t$ terms associated with the orbit characteristics

$$
\begin{gathered}
\frac{d z}{d t}=\frac{p_{z}}{\gamma m}, \\
\frac{d p_{z}}{d t}=-q\left(\frac{\partial \Phi}{\partial z}+\frac{1}{c} \frac{\partial A_{z}}{\partial t}\right) \\
\frac{d \mathbf{x}_{\perp}}{d t}=\frac{\mathbf{p}_{\perp}}{\gamma m},
\end{gathered}
$$

and

$$
\begin{aligned}
\frac{d \mathbf{p}_{\perp}}{d t}= & -\gamma_{b} m \omega_{\beta}^{2} \mathbf{x}_{\perp}-q \nabla_{\perp}\left(\Phi-\frac{v_{z}}{c} A_{z}\right) \\
& -\frac{q}{c}\left(\frac{\partial \mathbf{A}_{\perp}}{\partial t}+v_{z} \frac{\partial \mathbf{A}_{\perp}}{\partial z}\right)
\end{aligned}
$$

can cause numerical difficulties in particle simulations. We introduce here a novel way to eliminate this term by introducing the canonical momentum

$$
\mathbf{P}=\mathbf{p}+\frac{q}{c} \mathbf{A}
$$

as a new phase-space variable. From

$$
\frac{d \mathbf{A}}{d t} \approx \frac{\partial \mathbf{A}}{\partial t}+v_{z} \frac{\partial \mathbf{A}}{\partial z}
$$

we rewrite the orbit characteristics as

$$
\frac{d z}{d t}=\frac{1}{\gamma m}\left(P_{z}-\frac{q}{c} A_{z}\right)
$$

and

$$
\frac{d P_{z}}{d t}=-q \frac{\partial}{\partial z}\left(\Phi-\frac{v_{z}}{c} A_{z}\right)
$$

Thus, by transforming from $p_{z}$ to $P_{z}$, the time derivative of $A_{z}$ conveniently disappears from the equations of motion. Similarly, we obtain

$$
\frac{d \mathbf{x}_{\perp}}{d t}=\frac{1}{\gamma m}\left(\mathbf{P}_{\perp}-\frac{q}{c} \mathbf{A}_{\perp}\right)
$$

and

$$
\frac{d \mathbf{P}_{\perp}}{d t}=-\gamma_{b} m \omega_{\beta}^{2} \mathbf{x}_{\perp}-q \nabla_{\perp}\left(\Phi-\frac{v_{z}}{c} A_{z}\right)
$$

The Vlasov equation in the new coordinates can be expressed as

$$
\frac{d F}{d t} \equiv \frac{\partial F}{\partial t}+\frac{d \mathbf{x}}{d t} \cdot \frac{\partial F}{\partial \mathbf{x}}+\frac{d \mathbf{P}}{d t} \cdot \frac{\partial F}{\partial \mathbf{P}}=0,
$$

where the characteristics are defined by Eqs. (11) - (14). The corresponding form of Poisson's equation is

$$
\nabla^{2} \Phi=-4 \pi q \int F d \mathbf{P}
$$

and Ampere's law can be expressed as

$$
\left(\nabla^{2}-\frac{\omega_{p}^{2}}{c^{2}}\right) A_{z}=-\frac{4 \pi}{c} q \int \frac{P_{z}}{\gamma m} F d \mathbf{P}+\frac{1}{c} \frac{\partial}{\partial z}\left(\frac{\partial \Phi}{\partial t}\right)
$$

and

$$
\nabla^{2} \mathbf{A}_{\perp}=\frac{1}{c} \frac{\partial}{\partial t} \nabla_{\perp} \Phi
$$

where $\omega_{p}^{2} \equiv\left(4 \pi n q^{2} / m\right) \int F d \mathbf{P} / \gamma$ is the relativistic plasma frequency-squared. As before, the $\partial \Phi / \partial t$ term can 
then be calculated by the combination of Poisson's equation and the continuity equation, i.e.,

$$
\nabla^{2} \frac{1}{c} \frac{\partial \Phi}{\partial t}+\frac{\partial}{\partial z}\left(\frac{\omega_{p}^{2}}{c^{2}} A_{z}\right)=4 \pi q \frac{\partial}{\partial z} \int \frac{P_{z}}{\gamma m c} F d \mathbf{P} .
$$

Thus, Eqs. (11) - (19) constitute the Darwin model for an intense relativistic beam. In the long, thin-beam approximation, the terms associated with $\mathbf{A}_{\perp}$ and $\partial \Phi / \partial t$ in the equations of motion and Ampere's law can be dropped.

For a one-component relativistic beam traveling with a constant average velocity $\beta_{b} c$, which gives $A_{z} \simeq \beta_{b} \Phi$, the Darwin model in the laboratory frame becomes

$$
\begin{gathered}
\frac{\partial F}{\partial t}+\mathbf{v}_{\perp} \cdot \frac{\partial F}{\partial \mathbf{x}_{\perp}}+v_{z} \frac{\partial F}{\partial z} \\
-\left[\gamma_{b} m \omega_{\beta}^{2} \mathbf{x}_{\perp}+\frac{q}{\gamma_{b}^{2}} \nabla_{\perp} \Phi\right] \cdot \frac{\partial F}{\partial \mathbf{P}_{\perp}}-\frac{q}{\gamma_{b}^{2}} \frac{\partial \Phi}{\partial z} \frac{\partial F}{\partial P_{z}}=0
\end{gathered}
$$

and

$$
\nabla^{2} \Phi=-4 \pi q \int F d \mathbf{P}
$$

The single-beam Darwin model in the beam frame ('primed' variables) can be obtained by setting $\gamma_{b}=1$ and replacing $(\mathbf{x}, \mathbf{P}, t) \rightarrow\left(\mathbf{x}^{\prime}, \mathbf{p}^{\prime}, t^{\prime}\right)$. This gives

$$
\begin{gathered}
\frac{\partial F}{\partial t^{\prime}}+\mathbf{v}^{\prime} \cdot \frac{\partial F}{\partial \mathbf{x}^{\prime}}-\left[m \omega_{\beta}^{2} \mathbf{x}_{\perp}^{\prime}+q \nabla_{\perp}^{\prime} \Phi^{\prime}\right] \cdot \frac{\partial F}{\partial \mathbf{p}_{\perp}^{\prime}} \\
-q \frac{\partial \Phi^{\prime}}{\partial z^{\prime}} \frac{\partial F}{\partial p_{z}^{\prime}}=0
\end{gathered}
$$

where

$$
\nabla^{\prime 2} \Phi^{\prime}=-4 \pi q \int F d \mathbf{p}^{\prime}
$$

Thus, we recover the usual electrostatic model in the beam frame.

\section{NONLINEAR $\delta F$ DARWIN FORMALISM}

The corresponding $\delta f$ formalism $[10,11,12]$ in the laboratory frame can be derived by expressing $F=F_{0}+\delta f$, $\Phi=\Phi_{0}+\delta \Phi$ and $A_{z}=A_{z 0}+\delta A_{z}$, where $F_{0}$ satisfies

$$
\frac{\partial F_{0}}{\partial t}+\frac{d \mathbf{x}}{d t} \cdot \frac{\partial F_{0}}{\partial \mathbf{x}}+\left.\frac{d \mathbf{P}}{d t}\right|_{0} \cdot \frac{\partial F_{0}}{\partial \mathbf{P}}=0
$$

and $\left.\right|_{0}$ denotes the zeroth-order trajectories calculated by using the equilibrium potentials, $\Phi_{0}$ and $A_{z 0}$. The perturbed distribution is determined from

$$
\frac{d \delta f}{d t}=-\left.\frac{d \mathbf{P}_{\perp}}{d t}\right|_{\delta} \cdot \frac{\partial F_{0}}{d \mathbf{P}_{\perp}}-\left.\frac{d P_{z}}{d t}\right|_{\delta} \frac{\partial F_{0}}{\partial P_{z}},
$$

where $\left.\right|_{\delta}$ denotes the perturbed trajectories obtained from using the perturbed potentials, $\delta \Phi$ and $\delta A_{z}$. Defining $w=$ $\delta f / F$, the weight function evolves according to

$$
\frac{d w}{d t}=(1-w) \frac{1}{F_{0}} \frac{d \delta f}{d t} .
$$

In the Klimontovich-Dupree representation, the perturbed distribution is related to the particle weight through

$$
\delta f=\sum_{j=1}^{N} w_{j} \delta\left(\mathbf{x}-\mathbf{x}_{j}\right) \delta\left(\mathbf{P}-\mathbf{P}_{j}\right)
$$

where $N$ is the total number of particles in the simulation. The time evolution of $z_{j}, P_{z j}, \mathbf{x}_{\perp j}, \mathbf{P}_{\perp j}$, and $w_{j}$ for the $j$-th particle are described by Eqs. (11) - (14), and (25), respectively. For the field equations, the zeroth-order potentials, $\Phi_{0}$ and $A_{z 0}$, are obtained by using $F_{0}$ in Eqs. (16)(19) and the perturbed potentials, $\delta \Phi$ and $\delta A_{z}$, can be obtained by using $\delta f$.

\section{DISCUSSION}

The non-radiative Darwin model developed here for particle simulations is very similar to the usual electromagnetostatic model [12] and it is straightforward to implement. With the absence of the high-frequency radiation in the model, it has many numerical advantages over fully electromagnetic codes. The application of this model to the study of high-intensity beams will be reported elsewhere.

\section{ACKNOWLEDGMENTS}

The research was supported by the U.S. Department of Energy.

\section{REFERENCES}

[1] C. W. Nielson and H. R. Lewis, Methods in Computational Physics (Academic Press, New York, 1976), Vol. 16, p. 976.

[2] R. C. Davidson, D. A. Hammer, I. Haber, and C. E. Wagner, Phys. Fluids 15, 317 (1972).

[3] Busnardo-Neto et al., J. Comput. Phys. 23, 300 (1977).

[4] W. W. Lee, M. S. Chance and H. Okuda, Phys. Rev. Lett. 46, 1675 (1981).

[5] D. W. Hewett and J. K. Boyd, J. Comput. Phys. 70, 166 (1987).

[6] Sonnendrucker et al., J. Comput. Phys. 121, 218(1995).

[7] R. B. White and M. S. Chance, Phys. Fluids 27, 2455 (1984).

[8] R. G. Littlejohn, Phys. Fluids 27, 976 (1984).

[9] T. S. Hahm, W. W. Lee and A. Brizard, Phys. Fluids 31, 1940 (1987).

[10] S. E. Parker, and W. W. Lee, Phys. Fluids B 5, 77 (1993).

[11] W. W. Lee, Q. Qian, and R. C. Davidson, Phys. Lett. A 230, 347 (1997).

[12] H. Qin, R. C. Davidson, and W. W. Lee, Phys. Rev. Special Topics on Accelerators and Beams 3, 084401 (2000); 3, 109901 (2000).

[13] E. P. Lee, S. Yu, H. L. Buchanan, F. W. Chambers and M. N. Rosenbluth, Phys. Fluids 232095 (1980).

[14] C. G. Darwin, Philos. Mag. 39, 537 (1920).

[15] W. W. Lee, J. Comput. Phys. 72, 243 (1987). 